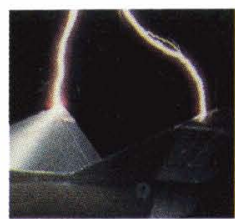

Public Safety

\section{Collision Avoidance System}

In the mid-1950s, when increasing air traffic caused congested skies over the U.S., government and aviation industry groups began a search for an airborne system to complement ground-based air traffic control and warn pilots of collision threats. A number of concepts were developed and tested over a span of more than two decades, but the technology of that era was not up to the complicated demands of collision warning.

By the 1980s, however, advancing technology permitted development of a commercially viable system, the Traffic Alert and Collision Avoidance System (TCAS II). In a concerted effort by the Federal Aviation Administration (FAA), the member airlines of the Air Transport Association, aircraft manufacturers, producers of avionic equipment and

TCAS is

designed to

alert pilots to

the presence

of other

aircraft in

their vicinity other aviation groups, TCAS II was thoroughly tested and specifications for its use in airline service were developed.

In 1989, following a Congressional mandate, the FAA ruled that TCAS would be required on all passenger carrying aircraft, including planes operated by U.S. flag airlines and those of foreign registry serving U.S. cities. Airline aircraft with more than 30 seats must have a TCAS II installed and operating by the end of 1993; passenger aircraft with 10 to 30 seats must have a TCAS by 1995 .

These rules were published after extensive TCAS II testing by several carriers in actual airline service. NASA's Ames Research Center played an important role in laying the groundwork for the airlines' inservice evaluation, teaming with the FAA to study the human performance factors associated with the use of TCAS II in an operational environment.

Using ground-based flight simulators flown by airline flight crews, Ames verified that pilots can accurately and quickly use TCAS II. The NASA program generated improvements in TCAS displays that resulted in both speed and accuracy increases
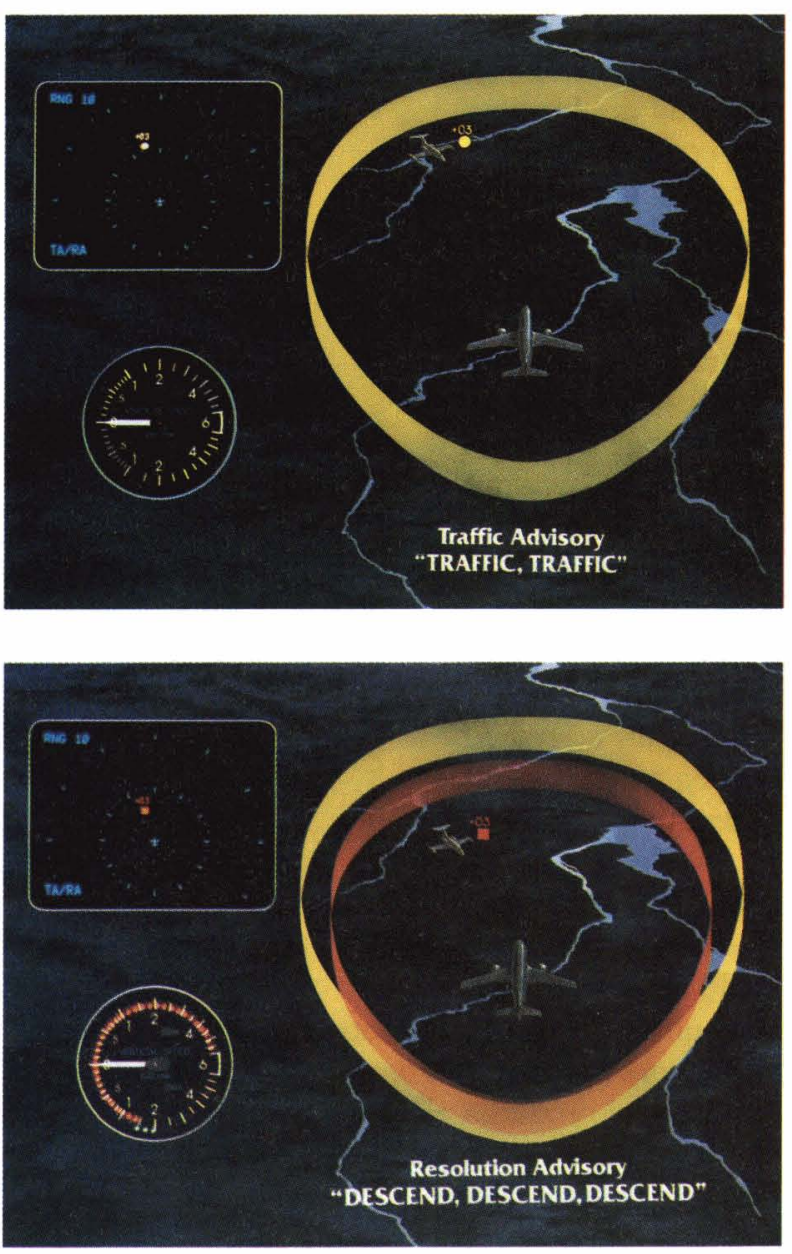

for the pilots' responses, and validated a set of pilot performance parameters that were coded in the system's logic for the best collision-evading maneuvers. Ames' work also contributed to development of airline pilot training procedures.

TCAS is designed to alert pilots to the presence of other aircraft in their vicinity, to identify and track "intruders" whose course and speed make them threats to safety, and to recommend action to avoid collision. In operation, the TCAS-equipped airplane sends a general interrogation signal to the transponders of other aircraft within the system's range. The transponders send reply signals to the TCAS aircraft, whose computer initiates a track for each replying aircraft, computes its range, bearing, altitude and other factors, and presents this information on a display in the cockpit. Display warnings are backed by automatic voice messages delivered through a cockpit speaker. 

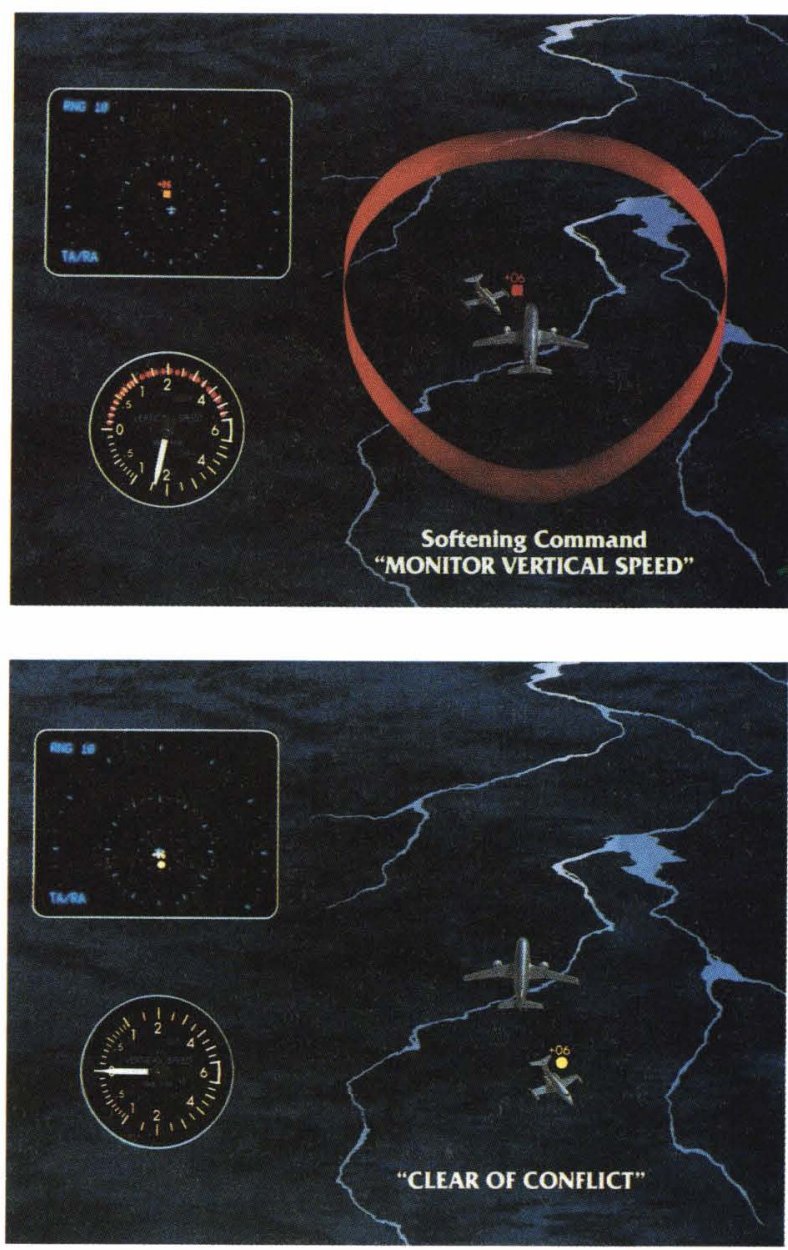

A display unit shows the TCAS aircraft as the center of the local airspace volume and indicates intruders in that airspace, as shown at upper left. Here an intruder has come close enough to trigger a Traffic Advisory. The computer changes the intruder's display symbol, previously a white diamond (non-threat) to a yellow circle (potential threat); the +03 adjacent to the circle means that the intruder is 300 feet higher than the TCAS aircraft. Simultaneously with the appearance of the yellow circle, the pilot hears the vocal Traffic Advisory "Traffic, traffic."

At lower left, the intruder has moved closer on a collision course and passed the inner ring on the display; it is now a definite collision threat. TCAS II issues a Resolution Advisory; it changes the intruder's symbol to red and the cockpit speaker delivers a vocal message announcing the required evasive action: "Descend, descend, descend." To find out how rapidly he must descend, the pilot consults the vertical speed indicator on his display, on which TCAS II has colored a portion of the dial red and green. The pilot must maneuver the airplane so that the needle moves out of the red area into the green target area; in this instance he must descend at 1,500 feet per minute.

The illustration at left shows his maneuver successful; there is now 600 feet of vertical separation between the two aircraft. Below, TCAS II indicates - by changing the intruder's symbol back to a yellow circle - that the crisis has passed; the voice message confirms "Clear of conflict."

Ames Research Center conducted three laboratory experiments to determine pilots' performance in responding to TCAS advisories. From the first study, Ames concludes that pilots were able to use TCAS II correctly within the allowable five-second response time and that TCAS was effective in reducing the severity of simulated traffic conflicts.

In the second experiment, Ames tested pilots' responses to changes in the avoidance advisories, for example, a second Resolution Advisory commanding an increase in the earlier-recommended climb or descent rate. Recorded reaction times suggested that pilots are able to respond to these changes within the targeted two seconds.

In the third experiment, pilot reactions to alternative TCAS displays were examined. For example, the vertical speed indicator was modified to include a green-lighted target vertical speed rate in addition to the red-lighted area. The results showed pilot preference to the red-and-green display over the red-only display and the TCAS II was changed accordingly. These studies contributed significantly to the airlines' evaluation of TCAS II and to improved air traffic system safety.
NASA studies

contributed

significantly

to the

airlines'

evaluation of

TCAS II and

to improved

air traffic

system safety 